\title{
Fatigue severity in anti-nuclear antibody- positive individuals does not correlate with pro-inflammatory cytokine levels or predict imminent progression to symptomatic disease
}

Waleed Hafiz ${ }^{1 \dagger}$, Rawad Nori ${ }^{1 \dagger}$, Ariana Bregasi ${ }^{2}$, Babak Noamani ${ }^{2}$, Dennisse Bonilla², Larissa Lisnevskaia ${ }^{3}$, Earl Silverman ${ }^{4}$, Arthur A. M. Bookman ${ }^{5}$, Sindhu R. Johnson ${ }^{1,6}$, Carolina Landolt-Marticorena ${ }^{2}$ and Joan Wither $2,5,7^{*}$ (iD

\begin{abstract}
Background: Fatigue is a common symptom of systemic autoimmune rheumatic disease (SARD). Patients with SARD have a protracted pre-clinical phase during which progressive immunologic derangements occur culminating in disease. In this study, we sought to determine when fatigue develops and whether its presence correlates with inflammatory factors or predicts disease progression.

Methods: Anti-nuclear antibody (ANA)-negative healthy controls (HCs) and ANA-positive participants with no criteria, at least one clinical criteria (undifferentiated connective tissue disease, UCTD), or meeting SARD classification criteria were recruited. Fatigue was assessed using a modified version of the FACIT-F questionnaire and the presence of fibromyalgia determined using a questionnaire based on the modified 2010 ACR criteria. Peripheral blood expression of five IFN-induced genes was quantified by NanoString and the levels of IL-1 $\beta$, IL-6, or TNF-a by ELISA.
\end{abstract}

Results: Fatigue was as prevalent and severe in individuals lacking SARD criteria as it was in UCTD and SARD. Overall, $\sim 1 / 3$ of $\mathrm{ANA}^{+}$subjects met fibromyalgia criteria, with no differences between sub-groups. Although fatigue was more severe in these individuals, those lacking fibromyalgia remained significantly more fatigued than ANA $^{-}$ HC. However, even in these subjects, fatigue correlated with the widespread pain index and symptom severity scores on the fibromyalgia questionnaire. Fatigue was not associated with elevated cytokine levels in any of the $\mathrm{ANA}^{+}$sub-groups and did not predict imminent disease progression.

Conclusions: Fatigue is common in $\mathrm{ANA}^{+}$individuals lacking sufficient criteria for a SARD diagnosis, correlates with fibromyalgia-related symptoms, and is not associated with inflammation or predictive of disease progression.

Keywords: Systemic autoimmune rheumatic disease, Fatigue, Cytokines

\footnotetext{
* Correspondence: Joan.Wither@uhnresearch.ca

${ }^{+}$Waleed Hafiz and Rawad Nori contributed equally to this work.

${ }^{2}$ Division of Genetics and Development, Krembil Research Institute, University

Health Network, 5KD402, 60 Leonard Avenue, Toronto, ON M5T 2S8, Canada

${ }^{5}$ Division of Rheumatology, Department of Medicine, Faculty of Medicine,

University Health Network, University of Toronto, Toronto, Canada

Full list of author information is available at the end of the article
}

(c) The Author(s). 2019 Open Access This article is distributed under the terms of the Creative Commons Attribution 4.0 International License (http://creativecommons.org/licenses/by/4.0/), which permits unrestricted use, distribution, and reproduction in any medium, provided you give appropriate credit to the original author(s) and the source, provide a link to the Creative Commons license, and indicate if changes were made. The Creative Commons Public Domain Dedication waiver (http://creativecommons.org/publicdomain/zero/1.0/) applies to the data made available in this article, unless otherwise stated. 


\section{Background}

Fatigue is a common feature of the anti-nuclear antibody (ANA)-positive systemic autoimmune rheumatic diseases (SARDs), including systemic lupus erythematosus (SLE), Sjogren's disease ( $\mathrm{SjD})$, systemic sclerosis (SSc), dermatomyositis, and mixed connective tissue disease [1-5]. It can be as disabling as other symptoms of organ dysfunction in these conditions and has a significant negative impact on the quality of life of affected patients [5-9]. Currently, the etiology of fatigue in SARD is poorly understood. Inflammation has been proposed to be a precipitating factor, but a lack of consistent findings showing that fatigue correlates with disease activity or that DMARDs and biologics significantly attenuate fatigue suggests that other factors, such as depression, pain, and poor sleep, contribute to its development [2-4, 6, 9-21].

One of the characteristic features of SARD is a prolonged pre-clinical phase during which autoantibodies are seen in the absence of symptoms [22, 23]. Although this is best established for SLE and SjD, it is likely that this also applies to other SARD. While this suggests that the presence of a positive ANA may predict eventual development of a SARD, $\sim 20 \%$ of healthy females have a positive ANA [24], the vast majority of which will not progress to SARD. In individuals who progress to a diagnosis of SLE, there is the insidious onset of accumulating clinical symptoms after a variable asymptomatic period [22]. This clinical course is likely also seen in other SARD, since it is not uncommon for individuals to present with insufficient symptoms/ signs to classify a SARD (termed undifferentiated connective tissue disease (UCTD)) and positive serologic findings, $\sim 20-40 \%$ of which go on to develop SARD in the next $3-5$ years [25-27].

Studies suggest that as patients progress from no symptoms to a diagnosis of SARD, there is a progressive increase in the levels as well as a change in the types of pro-inflammatory cytokines that are elaborated [28-30]. Given the proposed link between inflammation and fatigue, physicians are often concerned that the presence of profound fatigue in $\mathrm{ANA}^{+}$individuals may indicate the presence of unappreciated inflammation and a consequent increased risk of progression. In this study, we have addressed this question by examining fatigue in individuals who span the $\mathrm{ANA}^{+}$disease continuum from asymptomatic through UCTD to early SARD.

\section{Methods}

\section{Subjects and data collection}

$\mathrm{ANA}^{+}$individuals ( $\geq 1: 160$ by immunofluorescence), who had been referred to a clinic because of a recently discovered positive ANA, were consecutively recruited at the Toronto Western and Mount Sinai Hospitals. All patients were assessed by at least one of the participating rheumatologists and followed prospectively with clinical data being recorded through the use of standardized data collection forms. Participants were stratified into three groups based upon their initial assessment: (1) asymptomatic individuals, who lacked any clinical symptoms of SARD; (2) UCTD patients that had at least one clinical symptom of SARD but insufficient criteria to be classified as SARD; and (3) early SARD patients meeting classification criteria for a SARD (1997 ACR classification criteria for SLE [31], 2013 ACR-EULAR classification criteria for SSc [32], or the revised AmericanEuropean consensus criteria for $\mathrm{SjD}$ [33]) and that were within 2 years of diagnosis (except for $\mathrm{SjD}<5$ years). Subjects were excluded if they were on corticosteroids or DMARDS (except anti-malarials). All healthy controls (HCs) had their ANA and specific autoantibodies tested in the hospital laboratory to confirm that they were negative. $\mathrm{HC}$ with an ANA $\geq 1: 160$ were re-classified into the asymptomatic $\mathrm{ANA}^{+}$group, and those with a positive ANA < 1:160 or specific ANAs were excluded from the study. The study was approved by the Research Ethics Boards of both recruiting hospitals, and all participants signed informed consent.

\section{Clinical measures}

Fatigue was quantified using a modified version of the Functional Assessment Chronic Illness Therapy-Fatigue (FACIT-F) questionnaire with two questions that potentially might apply to disability rather than fatigue and one question regarding sleepiness in the day, a potential symptom of fibromyalgia, being removed [34]. To permit comparison with other studies using the FACIT$\mathrm{F}$, the score was calculated as 13 (the original number of questions on the FACIT-F) $\times$ the total score for answered questions divided by the number of questions answered. The presence of fibromyalgia was determined using a self-reported questionnaire using the modified 2010 ACR criteria [35, 36]. Patients were defined as having anemia if their hemoglobin level $<115 \mathrm{~g} / \mathrm{L}$, hypothyroidism if their $\mathrm{TSH}>5.5 \mathrm{mU} / \mathrm{L}$ and free $\mathrm{T} 4<11$ $\mathrm{pmol} / \mathrm{L}$, and depression if they were diagnosed by a physician and were on anti-depressant therapy.

\section{Measurement of autoantibodies}

Autoantibodies were measured in the University Health Network laboratory, with the ANA titer and pattern being determined by indirect immunofluorescence using HEp- 2 cells as a substrate. The levels of 11 specific autoantibodies (dsDNA, -chromatin, -Ro, -La, -Sm, -SmRNP, -RNP, -Jo-1, -Scl-70, -centromere, and ribosomal P) were assayed by the Bioplex ${ }^{\circ} 2200$ ANA Screening System (BioRad), using the company's cutoffs. 


\section{Cytokine measurement}

For measurement of interferon (IFN)-induced gene expression, total RNA was isolated from whole peripheral blood archived in Tempus tubes (Applied Biosystems) and gene expression was quantified by NanoString using a custom array (nanoString Technologies), as previously described [24]. $\log _{2}$ normalized expression levels of five IFN-induced genes (EPSTI1, IFI44L, LY6E, OAS3, RSAD2) were summed to generate a composite IFN5 score. Serum IFN- $\alpha$ and BAFF levels were measured by ELISA, as previously described [24], and serum IL-1- $\beta$, IL-6, and TNF- $\alpha$ levels using Quantikine High Sensitivity ELISA kits (R\&D Systems).

\section{Statistical analysis}

For comparisons of differences between three or more groups, a Kruskal-Wallis test was used followed by Dunns' post-test for multiple comparisons. When only two groups were compared, the Mann-Whiney $U$ test was performed for continuous variables and a $\chi^{2}$ or Fisher's exact test for discrete variables. The significance of association between variables was determined using Spearman's correlation coefficient. All statistical analyses were performed using GraphPad software (La Jolla, CA, USA).

\section{Results}

Fatigue is commonly seen in $\mathrm{ANA}^{+}$individuals lacking a SARD diagnosis and is often associated with symptoms of fibromyalgia

Demographics for the 146 study participants are summarized in Table 1. The majority of participants were female. Although the range of ages in each group was similar, the mean age for $\mathrm{HCs}$ was significantly lower than that for the three $\mathrm{ANA}^{+}$sub-groups $\left(\mathrm{ANA}^{+}\right.$no SARD symptoms (ANS), UCTD, SARD). Overall, $58 \%$ of

Table 1 Study participant characteristics

\begin{tabular}{|c|c|c|c|c|c|c|c|c|}
\hline & $\mathrm{HC}(n=29)$ & ANS $(n=46)$ & UCTD $(n=29)$ & $\operatorname{SARD}(n=42)$ & $\operatorname{SjD}(n=11)$ & $\operatorname{SLE}(n=11)$ & $\operatorname{SSc}(n=18)$ & MCTD/DM $(n=2)$ \\
\hline Age, mean $\pm S D$ & $29.3 \pm 9.8$ & $47.0 \pm 14.0$ & $48.3 \pm 16.5$ & $47.4 \pm 14.9$ & $47.7 \pm 14.4$ & $35.7 \pm 12.3$ & $54.7 \pm 13.3$ & $44 \pm 1.4$ \\
\hline Female sex, $n(\%)$ & $25(86.2)$ & $44(95.7)$ & $27(93.1)$ & $40(95.2)$ & $10(90.9)$ & $11(100)$ & $16(88.9)$ & $2(100)$ \\
\hline \multicolumn{9}{|l|}{ Ethnicity, n (\%) } \\
\hline Caucasian & $12(41.4)$ & $26(56.5)$ & $20(69.0)$ & $26(61.9)$ & $7(63.6)$ & $6(54.5)$ & $12(66.7)$ & $1(50)$ \\
\hline Asian & $0(0)$ & $3(6.5)$ & $5(17.2)$ & $2(4.8)$ & $1(9.1)$ & $0(0)$ & $1(5.6)$ & $0(0)$ \\
\hline South Asian & $5(17.2)$ & $5(10.9)$ & $2(6.9)$ & $5(11.9)$ & $2(18.2)$ & $1(9.1)$ & $2(11.1)$ & $0(0)$ \\
\hline Hispanic & $7(24.1)$ & $2(4.3)$ & $1(3.4)$ & $4(9.5)$ & $0(0)$ & $1(9.1)$ & $3(16.7)$ & $0(0)$ \\
\hline African Canadian & $1(3.4)$ & $7(15.2)$ & $0(0)$ & $1(2.4)$ & $0(0)$ & $1(9.1)$ & $0(0)$ & $0(0)$ \\
\hline Filipino & $1(3.4)$ & $1(2.2)$ & $0(0)$ & $2(4.8)$ & $0(0)$ & $1(9.1)$ & $0(0)$ & $1(50)$ \\
\hline Mixed & $3(10.3)$ & $2(4.3)$ & $1(3.4)$ & $2(4.8)$ & $1(9.1)$ & $1(9.1)$ & $0(0)$ & $0(0)$ \\
\hline Fibromyalgia, n (\%) & $0(0)$ & $17(37.0)$ & $13(44.8)$ & $12(28.6)$ & $2(18.2)$ & $3(27.3)$ & $6(33.3)$ & $1(50.0)$ \\
\hline Anemia, $n(\%)$ & $0(0)$ & $4(8.7)$ & $0(0)$ & $2(4.8)$ & $0(0)$ & $1(9.1)$ & $1(5.6)$ & $0(0)$ \\
\hline Hypothyroidism, n (\%) & $0(0)$ & $4(8.7)$ & $0(0)$ & $2(4.8)$ & $1(9.1)$ & $0(0)$ & $1(5.6)$ & $0(0)$ \\
\hline Depression, $n(\%)$ & $0(0)$ & $3(6.5)$ & $2(6.9)$ & $2(4.8)$ & $1(9.1)$ & $0(0)$ & $1(5.6)$ & $0(0)$ \\
\hline On anti-malarials, $n$ (\%) & $0(0)$ & $4(8.7)$ & $6(20.7)$ & $4(9.5)$ & $1(9.1)$ & $2(18.2)$ & $1(5.6)$ & $0(0)$ \\
\hline \multicolumn{9}{|l|}{ Specific antibodies, $n$ (\%) } \\
\hline dsDNA & $0(0)$ & $4(8.7)$ & $2(6.9)$ & $7(16.7)$ & $2(18.2)$ & $3(27.3)$ & $2(11.1)$ & $0(0)$ \\
\hline Ro & $0(0)$ & $11(23.9)$ & $9(31.0)$ & $19(45.2)$ & $11(100)$ & $5(45.5)$ & $3(16.7)$ & $0(0)$ \\
\hline La & $0(0)$ & $4(8.7)$ & $2(6.9)$ & $8(19.0)$ & $7(63.6)$ & $1(9.1)$ & $0(0)$ & $0(0)$ \\
\hline Sm & $0(0)$ & $2(4.3)$ & $1(3.4)$ & $4(9.5)$ & $0(0)$ & $3(27.3)$ & $0(0)$ & $1(50.0)$ \\
\hline $\mathrm{Sm} / \mathrm{RNP}$ & $0(0)$ & $3(6.5)$ & $2(6.9)$ & $6(14.3)$ & $0(0)$ & $4(36.4)$ & $1(5.6)$ & $1(50.0)$ \\
\hline RNP & $0(0)$ & $6(13.0)$ & $3(10.3)$ & $8(19.0)$ & $2(18.2)$ & $4(36.4)$ & $1(5.6)$ & $1(50.0)$ \\
\hline Scl-70 & $0(0)$ & $1(2.2)$ & $1(3.4)$ & $8(19.0)$ & $1(9.1)$ & $2(18.2)$ & $5(27.8)$ & $0(0)$ \\
\hline Jo-1 & $0(0)$ & $0(0)$ & $0(0)$ & $0(0)$ & $0(0)$ & $0(0)$ & $0(0)$ & $0(0)$ \\
\hline Centromere & $0(0)$ & $1(2.2)$ & $3(10.3)$ & 15 (35.7) & $0(0)$ & $1(9.1)$ & $13(72.2)$ & $1(50.0)$ \\
\hline Chromatin & $0(0)$ & $5(10.9)$ & $2(6.9)$ & $7(16.7)$ & $1(9.1)$ & $5(45.5)$ & $0(0)$ & $1(50.0)$ \\
\hline
\end{tabular}

Abbreviations: $H C$ healthy controls, ANS asymptomatic $\mathrm{ANA}^{+}$, UCTD undifferentiated connective tissue disease, SARD systemic autoimmune rheumatic disease, SjD Sjögren's disease, SLE systemic lupus erythematosus, SSC systemic sclerosis, MCTD mixed connective tissue disease, DM dermatomyositis, dsDNA double-stranded DNA, Sm Smith, RNP ribonuclear protein 
participants were Caucasian with a non-significant trend to fewer Caucasians in the $\mathrm{HC}$ group. A small number of $\mathrm{ANA}^{+}$participants were taking anti-malarials, including four individuals with ANS who had been started on anti-malarials prior to assessment in the clinic for symptoms that could not be definitely attributed to SARD (myalgia, arthralgia, and fatigue).

The presence of fatigue was determined using a modified version of the FACIT-F questionnaire, where lower scores indicate the presence of more fatigue. As shown in Fig. 1, all $\mathrm{ANA}^{+}$subjects regardless of the presence (SARD and UCTD) or absence of SARD symptoms/criteria (ANS) were significantly more fatigued than HCs, with no significant differences noted between the different $\mathrm{ANA}^{+}$sub-groups in the extent of fatigue. Using a cutoff of 3 SD below the mean for $\mathrm{ANA}^{-} \mathrm{HC}$ as significant fatigue, $67.4 \%$ of ANS, $79.3 \%$ UCTD, and $80.9 \%$ of SARD subjects were fatigued, as compared to $3.4 \%$ of ANA $^{-}$HC. Because many of the subjects suffered from fibromyalgia, and indeed this may have led to ANA testing in the case of ANS, we examined whether the fatigue was related to fibromyalgia, using the modified 2010 ACR criteria [35]. Individuals with a widespread pain index (WPI) of $\geq 7$ and a symptom severity (SS) score of $\geq 5$, or a WPI between 3 and 6 and a SS score $\geq 9$, on a self-administered questionnaire were considered to have fibromyalgia, which has been shown to have a sensitivity of $96.6 \%$ and specificity $91.8 \%$ for patients diagnosed clinically with fibromyalgia. Using this cutoff, none of the healthy controls and $37 \%$ of the $\mathrm{ANA}^{+}$subjects had fibromyalgia $(p<0.0001)$, with similar proportions of patients with fibromyalgia in each of the three $\mathrm{ANA}^{+}$ sub-groups (see Table 1). Not surprisingly, the FACIT-F scores were significantly lower in patients with fibromyalgia as compared to those without fibromyalgia and this was the case not only for the $\mathrm{ANA}^{+}$subjects as a whole (mean FACIT-F \pm SD, $35.5 \pm 12.2$ without fibromyalgia, $16.4 \pm 10.3$ with fibromyalgia, $p<0.0001$ ) but also for each of the $\mathrm{ANA}^{+}$sub-groups $(p<0.0001$, except UCTD $p=0.0026$ ) (Fig. 1). However, $\mathrm{ANA}^{+}$individuals without fibromyalgia still had significantly lower FACIT-F scores as compared to HC $(p<0.0001)$, and again this remained true for each of the $\mathrm{ANA}^{+}$subgroups (Fig. 1).

The WPI and SS scores derived from the fibromyalgia questionnaire represent a continuum that reflects the extent of pain and fatigue/somatic symptoms, respectively, independently of a diagnosis of fibromyalgia [36]. We therefore questioned whether the FACIT-F score correlated with these scores, even in the absence of fibromyalgia. As shown in Fig. 2, there was a strong negative correlation between the WPI and SS scores and the FACIT-F score in ANS, suggesting that the fatigue in these individuals may be related to symptoms of fibromyalgia. Of note, this was not simply due to redundancy between the questions being asked in the two questionnaires because only the SS score partially overlaps with the FACIT-F questionnaire, and equivalent strong correlations were seen for both WPI and SS subcomponents. While the correlations between the FACIT-F score and WPI and SS scores were somewhat weaker in UCTD and SARD patients lacking a fibromyalgia diagnosis (Fig. 2), there remained moderate negative correlations with the FACIT-F score, suggesting that in these patients as well a component of the fatigue may be due to fibromyalgia-related symptoms.

To further explore whether the fatigue in ANS individuals is predominantly related to symptoms of fibromyalgia, we compared the FACIT-F scores in the subset of $\mathrm{ANA}^{+}$subjects without SARD symptoms that had been recruited solely based upon their positive serology with those for HCs. These subjects included anti-Ro antibody-positive mothers who were referred for longitudinal follow-up after giving birth to a child with neonatal lupus or congenital heart block, and healthy controls re-classified to the ANS group following discovery of a positive ANA ( $\geq 1: 160)$ on laboratory testing. Nine subjects fulfilled these criteria, none of whom fulfilled criteria for fibromyalgia. As shown in Fig. 3, the FACIT-F scores for these subjects were significantly lower than those for the $\mathrm{ANA}^{-} \mathrm{HCs}$, despite WPI and SS scores that were roughly equivalent to HCs. This finding suggests that fatigue may be associated with a positive ANA and in support of this possibility an additional subject who was recruited as a $\mathrm{HC}$, who was found to have anti-Ro Abs but did not meet study criteria for inclusion in the $\mathrm{ANA}^{+}$subset, also had a low FACIT-F score $($ FACIT-F $=27.3)$. However, the impact of a positive ANA on fatigue appeared to be quite modest as compared to that of fibromyalgia-type symptoms.

As comorbidities, such as anemia, hypothyroidism, or depression, have been shown to contribute to chronic fatigue [34, 37-39], we assessed whether fatigue was more profound in $\mathrm{ANA}^{+}$subjects with these diagnoses. Very few of the subjects had these comorbidities (Table 1), and no significant differences were seen in the FACIT-F scores between subjects with and without these conditions (data not shown, all $p>0.05$ ).

\section{The presence of fatigue does not correlate with inflammation in any of the ANA ${ }^{+}$sub-groups}

Consistent with the possibility that fatigue in SARD results from inflammation, some studies have found a correlation with disease activity and/or reductions in fatigue following treatment with DMARDs or biologics [2, 11, $15,17,18,21]$. Of the pro-inflammatory cytokines that are typically elevated in SARD, IL-1 $\beta$, IL-6, and TNF- $\alpha$, in particular, have been linked to fatigue [40-42]. 


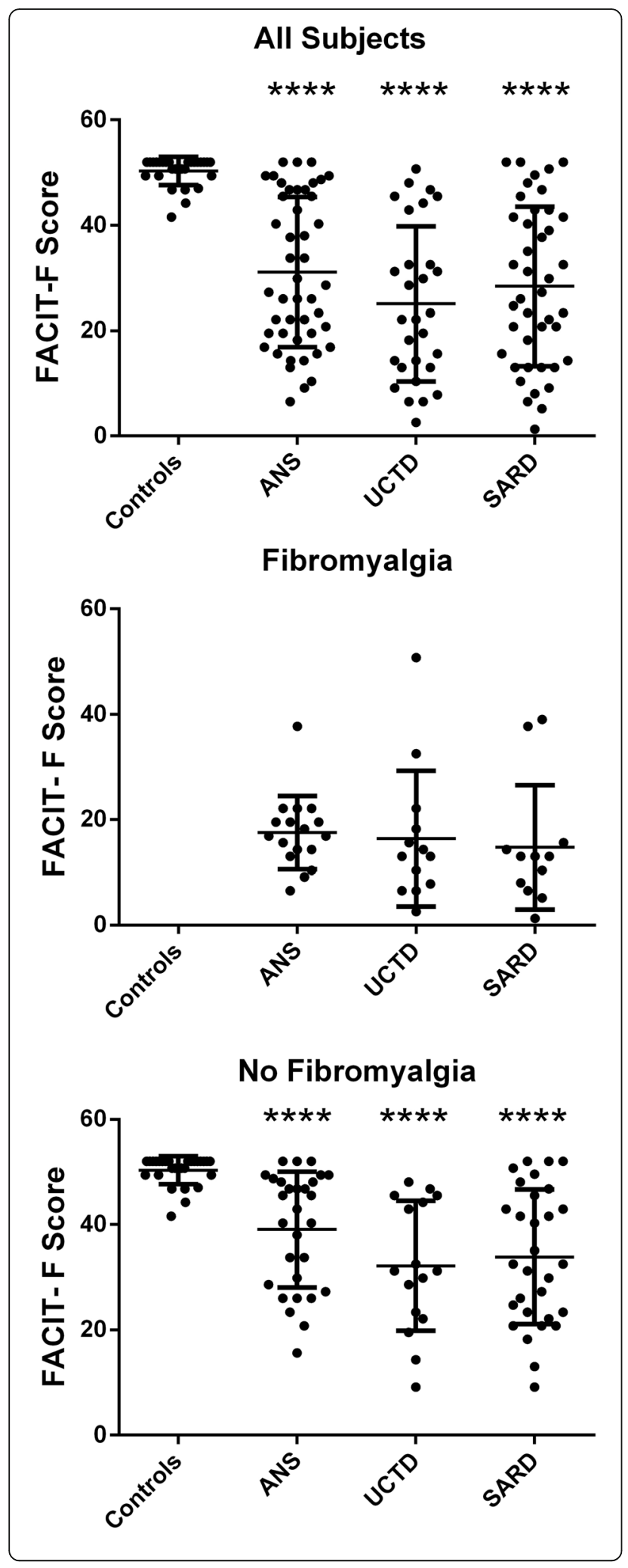

Fig. 1 Asymptomatic $\mathrm{ANA}^{+}$individuals lacking a SARD diagnosis have just as severe fatigue as UCTD and SARD patients. Shown are scatterplots with results for all subjects and subdivided into those with and without a diagnosis of fibromyalgia. Columns indicate results for $\mathrm{ANA}^{-}$healthy controls (Controls), asymptomatic $\mathrm{ANA}^{+}$ individuals (ANS), and patients with UCTD or SARD. Every data point corresponds to an individual subject, with the bars representing the mean with SD. For each set of comparisons, statistical significance was determined using the Kruskal-Wallis test with Dunn's post-test for multiple comparisons, as compared to controls. ${ }^{*} p \leq 0.05$,

${ }^{* *} p \leq 0.01,{ }^{* * *} p \leq 0.001,{ }^{* * *} p \leq 0.0001$. There were no significant differences between the different $\mathrm{ANA}^{+}$sub-groups

As outlined previously, there was no association between the FACIT-F score and the presence or absence of SARD symptoms/signs in $\mathrm{ANA}^{+}$subjects (see Fig. 1) nor was there an association between ANA titer or the number of different ANA specificities as measured by the Bioplex ANA screen and fatigue (data not shown). To examine the association between fatigue and inflammation, we quantified the levels of type I IFN-induced gene expression as well as the serum levels of IL-1 $\beta$, IL6 , and TNF- $\alpha$. We have previously shown that a significant proportion of $\mathrm{ANA}^{+}$individuals have elevated type I IFN levels including those without SARD symptoms/ signs and that these elevations correlate with the levels of several IFN-driven cyto/chemokines, such as BAFF [24]. Similar elevations of IFN-induced gene expression were seen in the $\mathrm{ANA}^{+}$individuals that were examined in this study (some of which overlapped with those previously published, Fig. 4), which did not correlate with fatigue (Table 2). As IL-1 $\beta$ was not significantly elevated in any of the $\mathrm{ANA}^{+}$groups when compared to $\mathrm{HC}$, and given that the levels of IL- $\beta$ in $>50 \%$ of the samples were below the limit of detection of the ELISA, associations with this cytokine were not examined further. A trend to increased levels of IL- 6 and TNF- $\alpha$ was seen in all $\mathrm{ANA}^{+}$groups as compared to $\mathrm{HC}$, which was most pronounced in SARD. This achieved statistical significance only for TNF- $\alpha$ in ANS and SARD patients. As shown in Table 2, there was no association between fatigue and any of the cytokines, either for $\mathrm{ANA}^{+}$individuals as a whole or for any of the $\mathrm{ANA}^{+}$sub-groups, and similar negative findings were seen when patients with and without fibromyalgia were examined independently (Additional file 1: Table S1).

To further explore the association between fibromyalgia, pain, fatigue, and inflammation, we examined the correlation between cytokine levels and the presence or absence of fibromyalgia, as well as the WPI and SS scores. There were no significant differences in the levels of cytokines between $\mathrm{ANA}^{+}$individuals with or without at fibromyalgia diagnosis (data not shown). However, there was a significant correlation between IL-6 levels and the WPI for the $\mathrm{ANA}^{+}$individuals as a whole, which 

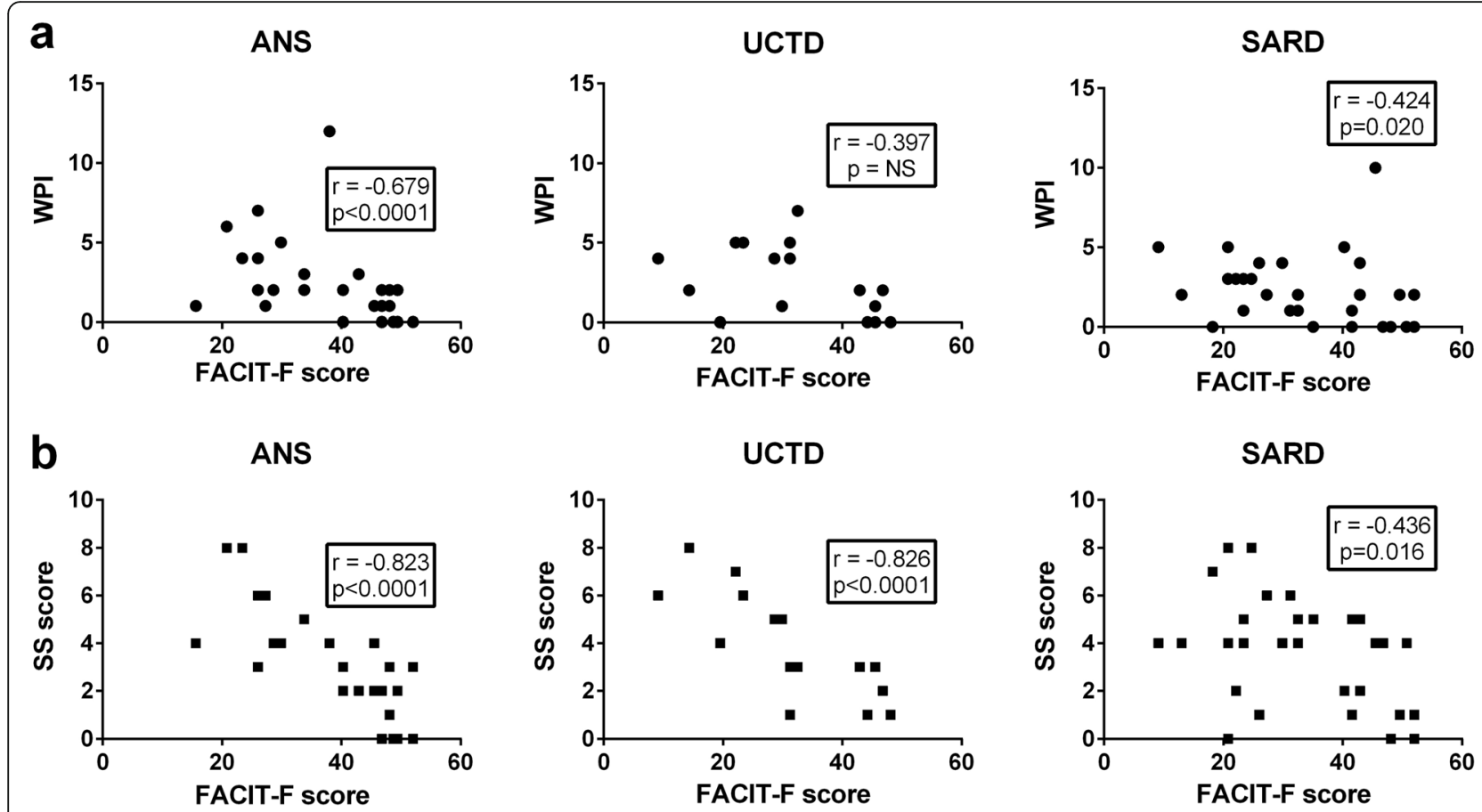

Fig. 2 Correlations between fatigue and the widespread pain index (WPI) or symptom severity (SS) scores on the fibromyalgia questionnaire for the subjects without a fibromyalgia diagnosis. Results have been stratified into the different $\mathrm{ANA}^{+}$sub-groups (asymptomatic ANA ${ }^{+}$individuals (ANS), UCTD, and SARD). a Correlation between the WPI score and FACIT-F score. $\mathbf{b}$ Correlation between the SS score and FACIT-F score. Every symbol corresponds to an individual subject. Values in the boxes show the Spearman correlation coefficient and significance of association

remained marginally significant in the ANS and SARD sub-groups (Table 2). In general, the strength of this association was greater than that seen for IL-6 levels with the SS score (with the exception of the UCTD subgroup, see Table 2) and also was greater for individuals without a fibromyalgia diagnosis as compared to those with a fibromyalgia diagnosis (Additional file 1: Table S2). These findings suggest that the association of IL-6 levels with WPI may be independent of fatigue and instead may indicate that a component of the arthralgia results from inflammation. In support of this concept, there was also a significant association between TNF- $\alpha$ levels and the WPI in $\mathrm{ANA}^{+}$subjects without fibromyalgia, which was largely driven by the SARD sub-group.

\section{Severe fatigue does not predict imminent progression to SARD}

Physicians are often concerned that the presence of profound fatigue in $\mathrm{ANA}^{+}$individuals might indicate an increased likelihood of progression to a UCTD or SARD. To investigate this possibility, we contrasted fatigue in patients who demonstrated progression, as indicated by the development of new SARD classification criteria, as compared to those who did not. There were 3 of the 26 ANS patients with at least 1 year of follow-up who developed definitive SARD criteria (1 developed seropositive rheumatoid arthritis, 1 had the development of new lupus-associated autoantibodies and Raynaud's phenomenon, and 1 developed arthritis, rash, and Raynaud's phenomenon fulfilling classification criteria for SLE). There was a non-statistically significant trend to less fatigue in progressors compared to non-progressors (median FACIT-F: progressors 46.8, non-progressors $26, p=0.150$ ). Decreases were also seen in the WPI and SS scores for progressors, which achieved statistical significance for the SS score $(p=$ 0.031). Consistent with a recently published study suggesting that $\mathrm{ANA}^{+}$individuals lacking a SARD diagnosis with high IFN scores are more likely to progress to SARD than those with low IFN scores, the IFN scores in progressors were significantly higher than in nonprogressors $(p=0.0054)$. No differences were seen in the levels of IL- 6 and TNF-a between progressors and non-progressors.

Similar but less pronounced findings were observed for patients with UCTD. Four of 22 UCTD patients progressed in a 1-year follow-up period, with development of new SARD criteria (1 new onset arthritis) or evolution to SARD (2 SjD, $1 \mathrm{SSc}$ ). Although there were trends to decreased fatigue, WPI, and SS scores, as well as increased IFN scores in progressors as compared to nonprogressors, these did not achieve statistical significance. 


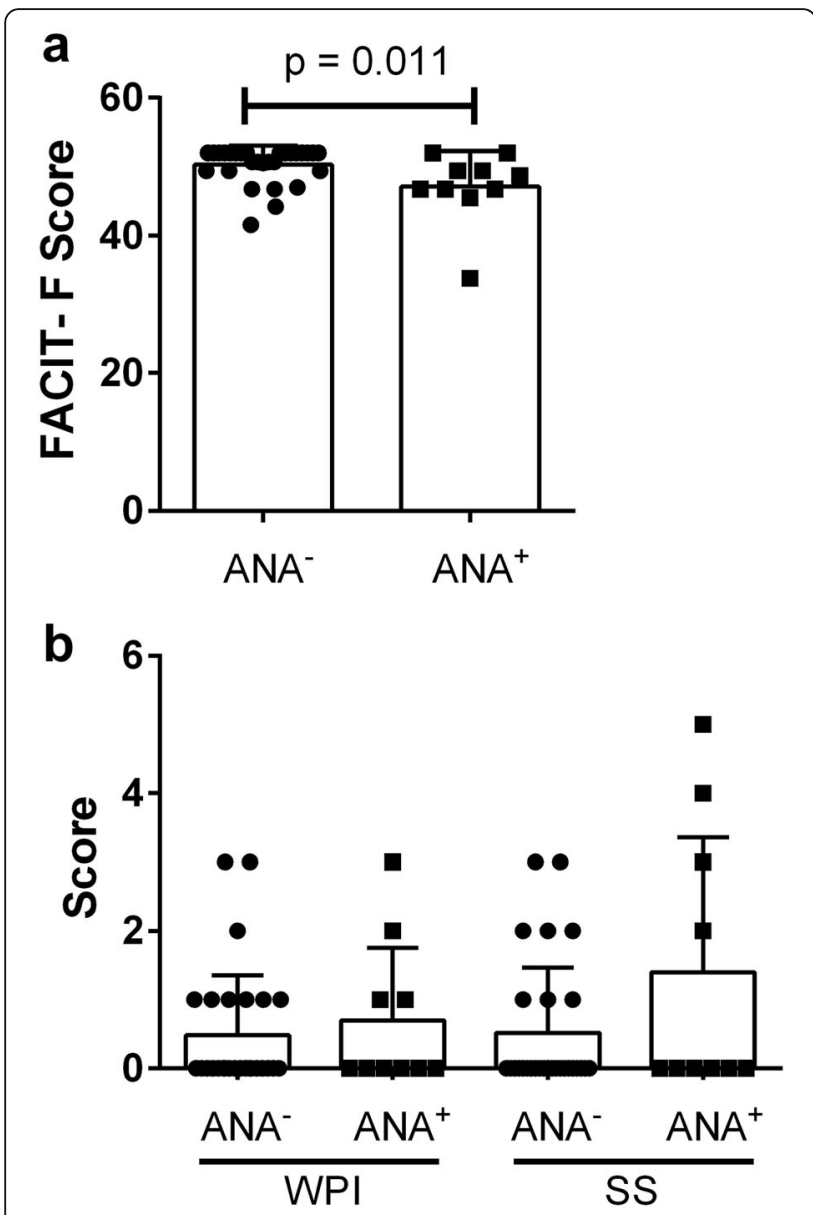

Fig. 3 Presence of mild fatigue in $\mathrm{ANA}^{+}$individuals who were recruited as healthy controls or who gave birth to a baby with neonatal lupus. a Fatigue, as measured by the FACIT-F score, and $\mathbf{b}$ WPI and SS scores, as measured by the fibromyalgia questionnaire, in $\mathrm{ANA}^{-}$healthy controls $\left(\mathrm{ANA}^{-}\right.$) and $\mathrm{ANA}^{+}$individuals, as outlined above $\left(\mathrm{ANA}^{+}\right)$. Every symbol corresponds to an individual subject with bars indicating the mean with SD. Significant differences are shown and were calculated using the Mann-Whitney $U$ test comparing $\mathrm{ANA}^{-}$and $\mathrm{ANA}^{+}$subjects

\section{Discussion}

In this study, we show that the prevalence and severity of fatigue in ANS is similar to that seen in UCTD and early SARD and comparable to that seen in previous studies of $\mathrm{ANA}^{+}$SARD where the FACIT-F was used to quantify fatigue $[8,9,43]$. As noted in other studies of SARD, a substantial component of this fatigue was related to fibromyalgia [44-46], which was present in $\sim 1 /$ 3 of all $\mathrm{ANA}^{+}$subjects regardless of the presence or absence of SARD criteria, and which was associated with significantly more marked fatigue as measured by the FACIT-F than seen in subjects lacking fibromyalgia. Nevertheless, even $\mathrm{ANA}^{+}$subjects lacking fibromyalgia were still significantly more fatigued than $\mathrm{ANA}^{-} \mathrm{HC}$ and the severity of the fatigue was again similar in ANS to that observed for UCTD and SARD patients.
In ANS lacking fibromyalgia, there remained a strong correlation between the WPI and SS scores and the FACIT-F, suggesting that although these patients did not meet criteria they may still have had fibromyalgialike symptoms. This may reflect a selection bias, where individuals with pain and fatigue are more likely to seek medical care and have serologic testing performed, in part due to the perception that these symptoms may be a surrogate for ongoing inflammation. However, similar but slightly weaker correlations were also seen for UCTD and early SARD patients, indicating that even in individuals who have SARD criteria, a significant component of their fatigue may be due to fibromyalgia-like symptoms. The findings are in keeping with previous studies showing a correlation between fibromyalgia, disturbances of sleep, tender points, or pain and fatigue in SARD $[6,16,45,46]$.

We recognize that the diagnostic criteria that we used for fibromyalgia were developed and validated for patients without inflammatory rheumatic disease. However, we used these in UCTD and SARD patients to enable comparison with $\mathrm{ANA}^{-} \mathrm{HC}$ and ANS subjects and because the majority of our patients lacked inflammatory arthritis. Of the 12 SARD patients that met diagnostic criteria for fibromyalgia, only 3 had tender joints thought to be related to inflammatory arthritis, with only one having swollen joints. Notably, all 3 of these patients had more generalized pain on their fibromyalgia questionnaire than could be accounted for by their tender joints. In fact, the majority of SARD patients (9/12) that met fibromyalgia criteria had a WPI $\geq 7$. Thus, SARD patients did not solely meet fibromyalgia criteria based upon their fatigue symptoms, but also had substantial unexplained generalized pain consistent with this diagnosis. These findings are compatible with previous studies that found an increased prevalence of fibromyalgia in SARD patients, either when diagnosed using conventional clinical criteria or the diagnostic criteria used in this study [47-50].

The close correlation between fatigue and fibromyalgialike symptoms in $\mathrm{ANA}^{+}$individuals that are referred to a rheumatologist lacking clinical SARD diagnostic criteria made it difficult to assess whether the presence of an ANA alone was associated with fatigue. We circumvented this problem by examining ANS who had been recruited as $\mathrm{HC}$ or whose ANA was discovered following delivery of a baby with neonatal lupus. Although fibromyalgia-like symptoms in these individuals were no more prevalent than in $\mathrm{ANA}^{-} \mathrm{HC}$, they were statistically significantly more fatigued. However, the magnitude of this difference was small and the severity of this fatigue was very mild, suggesting that the majority of the fatigue seen in the ANS individuals referred to rheumatologists is unrelated to the immunologic derangement that produces a positive 


\section{Type I IFN}

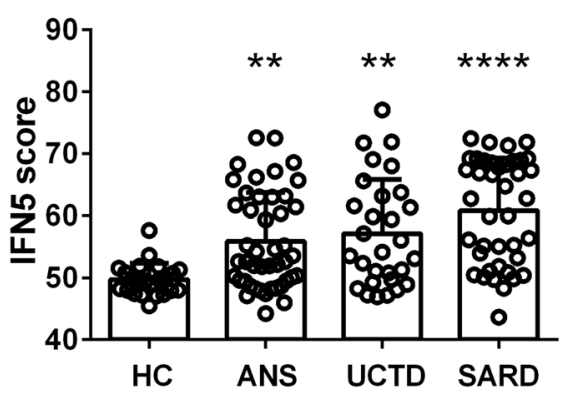

IL-6

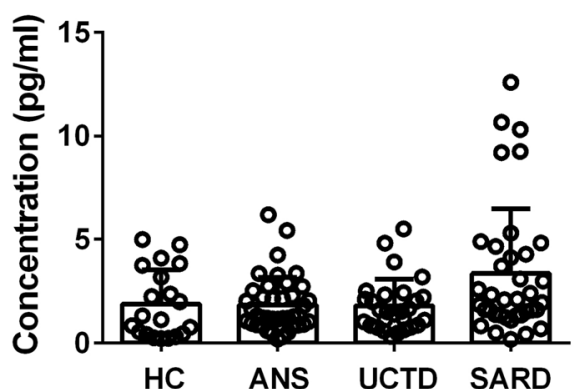

IL-1 $\beta$

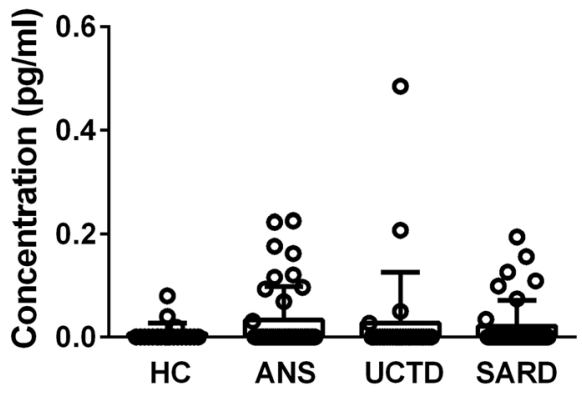

TNF- $\alpha$

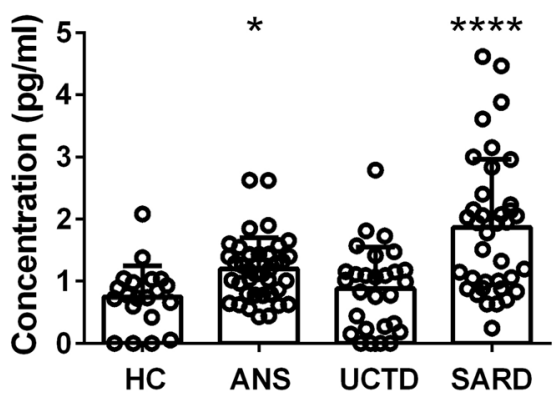

Fig. 4 Levels of selected pro-inflammatory cytokines in $\mathrm{ANA}^{+}$individuals stratified based upon the presence of clinical SARD diagnostic criteria. Columns indicate results for $\mathrm{ANA}^{-}$healthy controls (HC), ANA ${ }^{+}$individuals lacking any SARD clinical diagnostic criteria (ANS), and patients with UCTD or SARD. Every data point corresponds to an individual subject, with the bars representing the mean with SD. For each set of comparisons, statistical significance was determined using the Kruskal-Wallis test with Dunn's post-test for multiple comparisons, as compared to HC. ${ }^{*} p \leq 0.05$, ${ }^{* *} p \leq 0.01,{ }^{* * *} p \leq 0.001,{ }^{* * *} p \leq 0.0001$

ANA. In support of this concept, no correlation was seen between ANA titer or number of different ANA specificities and the extent of fatigue.

The contribution of inflammation to fatigue in rheumatic diseases remains unclear. Studies showing that injection of some of the key cytokines produced in rheumatic diseases into $\mathrm{HC}$, such as IL- $1 \beta$ or IL-6, produces fatigue and that biologics targeting IL- 6 or TNF- $\alpha$ ameliorate fatigue [11, 40-42] suggest a role for these molecules in the development of fatigue. However, while the levels of these cytokines tend to correlate with disease activity, very few studies have shown an association between

Table 2 Associations with inflammatory cytokines

\begin{tabular}{|c|c|c|c|c|}
\hline Cytokine & All ANA,$+ \rho$ & ANS, $\rho$ & UCTD, $\rho$ & SARD, $\rho$ \\
\hline \multicolumn{5}{|l|}{ A. FACIT-F } \\
\hline Type I IFN & 0.071 & 0.118 & 0.213 & -0.029 \\
\hline IL-6 & -0.072 & -0.092 & -0.224 & 0.015 \\
\hline TNF-a & -0.101 & -0.093 & -0.135 & -0.152 \\
\hline \multicolumn{5}{|l|}{ B. WPI } \\
\hline Type I IFN & -0.064 & -0.142 & -0.038 & -0.022 \\
\hline IL-6 & $0.239(0.014)^{*}$ & $0.308(0.053)$ & 0.211 & $0.288(0.084)$ \\
\hline TNF-a & 0.090 & 0.258 & 0.083 & 0.201 \\
\hline \multicolumn{5}{|l|}{ C. SS score } \\
\hline Type I IFN & $-0.164(0.081)$ & -0.132 & -0.204 & -0.076 \\
\hline IL-6 & $0.174(0.075)$ & 0.223 & $0.397(0.036)$ & 0.090 \\
\hline TNF-a & -0.005 & 0.089 & 0.057 & 0.011 \\
\hline
\end{tabular}

*Significant differences are indicated in italics with the $p$ value shown in the brackets. Any $p$ values less than 0.1 are also shown as indicative of trends 
disease activity and fatigue [2, 15, 17]. Furthermore, where these cytokines have been measured, no correlation has been noted $[14,19,20]$. In this study, we show that although the levels of TNF- $\alpha$ are significantly elevated in SARD and ANS, and there is a trend to increased IL-6 in these groups, there was no correlation with fatigue, confirming previous studies of SARD [6, $10,12,16]$ and indicating that this extends to individuals with ANS and UCTD. However, some correlations were seen for these cytokines with the WPI. Since the majority of patients did not suffer from joint inflammation even within the SARD group, it is possible that these associations reflect the ability of IL- 6 and TNF- $\alpha$ to stimulate nociceptive sensory neurons leading to enhanced pain sensitivity [51].

We and others have previously shown that elevated levels of type I IFN are associated with symptomatic progression in ANS and UCTD [52, 53]. Here, we show that there is no association between type I IFN levels and fatigue and that fatigue does not predict symptomatic progression. Indeed, there was a non-significant trend to less fatigue in progressors.

\section{Conclusions}

Our findings have important clinical implications. Firstly, clinicians can reassure their fatigued ANS patients that their fatigue does not indicate that they are at increased risk for imminent progression; secondly, the presence of significant fatigue should not prompt initiation of treatment with DMARDs; and thirdly, our findings suggest that treatments that have been shown to improve fatigue, such as exercise programs, promotion of good sleep hygiene, addressing life stressors and depression [54], or drug therapy for fibromyalgia, may be more appropriate therapies for these individuals.

\section{Supplementary information}

Supplementary information accompanies this paper at https://doi.org/10. 1186/s13075-019-2013-9

Additional file 1: Table S1. Correlations between the FACIT-F score and inflammatory cytokines in $\mathrm{ANA}^{+}$subjects. Table S2. Correlations between the WPI and inflammatory cytokines in $\mathrm{ANA}^{+}$subjects.

\section{Abbreviations}

ANA: Anti-nuclear antibody; SARD: Systemic autoimmune rheumatic disease; SLE: Systemic lupus erythematosus; SjD: Sjogren's disease; SSc: Systemic sclerosis; UCTD: Undifferentiated connective tissue disease; HC: Healthy control; FACIT-F: Functional Assessment Chronic Illness Therapy-Fatigue; IFN: Interferon; ANS: ANA ${ }^{+}$no SARD symptoms; WPI: Widespread pain index; SS: Symptom severity

\section{Acknowledgements}

Not applicable.

\section{Authors' contributions}

$A A M B, S R J, C L-M$, and JW were responsible for study conception and design. $W H, R N, A B, B N, D B, L L, E S, A A M B, S R J, C L-M$, and JW were responsible for the acquisition of data. $\mathrm{WH}, \mathrm{RN}$, and JW performed the data analysis and interpretation. All authors were involved in drafting the article or revising it critically for important intellectual content, and all authors approved the final version to be published.

\section{Funding}

This work was supported by a Strategic Operating Grant from The Arthritis Society of Canada [grant number SOG-15-281]. SRJ is supported by a CIHR Clinician Scientist Award, the Oscar and Eleanor Markovitz Fund for Scleroderma Research, and the Freda Fejer Fund for Scleroderma Research. CL-M was the recipient of a Clinician-Scientist Salary Award from the Arthritis and Autoimmunity Research Centre of the University Health Network. JW is funded by The Arthritis Centre of Excellence of the University of Toronto and is the recipient of a Department of Medicine Merit Award.

Availability of data and materials

Supporting data is located in Additional file 1.

Ethics approval and consent to participate

The study was approved by the Research Ethics Boards of the University Health Network (12-5455-BE) and Mount Sinai Hospital, and all participants signed informed consent.

Consent for publication

Not applicable.

\section{Competing interests}

The authors declare that they have no competing interests.

\section{Author details}

${ }^{1}$ Division of Rheumatology, Department of Medicine, Faculty of Medicine, University Health Network and Mount Sinai Hospital, University of Toronto, Toronto, Canada. ${ }^{2}$ Division of Genetics and Development, Krembil Research Institute, University Health Network, 5KD402, 60 Leonard Avenue, Toronto, ON M5T 2S8, Canada. ${ }^{3}$ Lakeridge Health Services, Oshawa, Canada. ${ }^{4}$ Division of Rheumatology, Hospital for Sick Children, Department of Pediatrics, Faculty of Medicine, University of Toronto, Toronto, Canada. ${ }^{5}$ Division of Rheumatology, Department of Medicine, Faculty of Medicine, University Health Network, University of Toronto, Toronto, Canada. ${ }^{6}$ Institute of Health Policy, Management and Evaluation, University of Toronto, Toronto, Canada. ${ }^{7}$ Department of Immunology, Faculty of Medicine, University of Toronto, Toronto, Canada.

Received: 3 July 2019 Accepted: 21 September 2019

Published online: 04 November 2019

References

1. Barendregt PJ, Visser MR, Smets EM, Tulen $J H$, van den Meiracker AH, Boomsma F, Markusse HM. Fatigue in primary Sjogren's syndrome. Ann Rheum Dis. 1998;57(5):291-5.

2. Tench CM, McCurdie I, White PD, D'Cruz DP. The prevalence and associations of fatigue in systemic lupus erythematosus. Rheumatology (Oxford). 2000;39(11):1249-54.

3. Thombs BD, Taillefer SS, Hudson M, Baron M. Depression in patients with systemic sclerosis: a systematic review of the evidence. Arthritis Rheum. 2007;57(6):1089-97.

4. Segal B, Thomas W, Rogers T, Leon JM, Hughes P, Patel D, Patel K, Novitzke J. Rohrer M, Gopalakrishnan R, et al. Prevalence, severity, and predictors of fatigue in subjects with primary Sjogren's syndrome. Arthritis Rheum. 2008; 59(12):1780-7.

5. Bassel M, Hudson M, Taillefer SS, Schieir O, Baron M, Thombs BD. Frequency and impact of symptoms experienced by patients with systemic sclerosis: results from a Canadian National Survey. Rheumatology (Oxford). 2011;50(4):762-7.

6. Bruce IN, Mak VC, Hallett DC, Gladman DD, Urowitz MB. Factors associated with fatigue in patients with systemic lupus erythematosus. Ann Rheum Dis. 1999:58(6):379-81.

7. Schmeding A, Schneider M. Fatigue, health-related quality of life and other patient-reported outcomes in systemic lupus erythematosus. Best Pract Res Clin Rheumatol. 2013;27(3):363-75. 
8. Segal B, Bowman SJ, Fox PC, Vivino FB, Murukutla N, Brodscholl J, Ogale S, McLean L. Primary Sjogren's syndrome: health experiences and predictors of health quality among patients in the United States. Health Qual Life Outcomes. 2009:7:46.

9. Strickland G, Pauling J, Cavill C, McHugh N. Predictors of health-related quality of life and fatigue in systemic sclerosis: evaluation of the EuroQol-5D and FACIT-F assessment tools. Clin Rheumatol. 2012;31(8):1215-22.

10. Burgos PI, Alarcon GS, McGwin G Jr, Crews KQ, Reveille JD, Vila LM. Disease activity and damage are not associated with increased levels of fatigue in systemic lupus erythematosus patients from a multiethnic cohort: LXVII. Arthritis Rheum. 2009;61(9):1179-86.

11. Illei GG, Shirota Y, Yarboro CH, Daruwalla J, Tackey E, Takada K, Fleisher T, Balow JE, Lipsky PE. Tocilizumab in systemic lupus erythematosus: data on safety, preliminary efficacy, and impact on circulating plasma cells from an open-label phase I dosage-escalation study. Arthritis Rheum. 2010;62(2):542-52.

12. Jump RL, Robinson ME, Armstrong AE, Barnes EV, Kilbourn KM, Richards HB. Fatigue in systemic lupus erythematosus: contributions of disease activity, pain, depression, and perceived social support. J Rheumatol. 2005:32(9): 1699-705.

13. McKinley PS, Ouellette SC, Winkel GH. The contributions of disease activity, sleep patterns, and depression to fatigue in systemic lupus erythematosus. A proposed model. Arthritis Rheum. 1995;38(6):826-34.

14. Omdal R, Mellgren SI, Koldingsnes W, Jacobsen EA, Husby G. Fatigue in patients with systemic lupus erythematosus: lack of associations to serum cytokines, antiphospholipid antibodies, or other disease characteristics. J Rheumatol. 2002;29(3):482-6.

15. Tayer WG, Nicassio PM, Weisman MH, Schuman C, Daly J. Disease status predicts fatigue in systemic lupus erythematosus. J Rheumatol. 2001;28(9): 1999-2007.

16. Wang B, Gladman DD, Urowitz MB. Fatigue in lupus is not correlated with disease activity. J Rheumatol. 1998;25(5):892-5.

17. Wysenbeek AJ, Leibovici L, Weinberger A, Guedj D. Fatigue in systemic lupus erythematosus. Prevalence and relation to disease expression. $\mathrm{Br}$ J Rheumatol. 1993;32(7):633-5.

18. Dass S, Bowman SJ, Vital EM, Ikeda K, Pease CT, Hamburger J, Richards A, Rauz S, Emery P. Reduction of fatigue in Sjogren syndrome with rituximab: results of a randomised, double-blind, placebo-controlled pilot study. Ann Rheum Dis. 2008;67(11):1541-4.

19. Hartkamp A, Geenen R, Bijl M, Kruize AA, Godaert GL, Derksen RH. Serum cytokine levels related to multiple dimensions of fatigue in patients with primary Sjogren's syndrome. Ann Rheum Dis. 2004;63(10):1335-7.

20. Howard Tripp N, Tarn J, Natasari A, Gillespie C, Mitchell S, Hackett KL, Bowman SJ, Price E, Pease CT, Emery P, et al. Fatigue in primary Sjogren's syndrome is associated with lower levels of proinflammatory cytokines. RMD Open. 2016;2(2):e000282.

21. Meijer JM, Meiners PM, Vissink A, Spijkervet FK, Abdulahad W, Kamminga N, Brouwer E, Kallenberg CG, Bootsma H. Effectiveness of rituximab treatment in primary Sjogren's syndrome: a randomized, double-blind, placebocontrolled trial. Arthritis Rheum. 2010;62(4):960-8.

22. Arbuckle MR, McClain MT, Rubertone MV, Scofield RH, Dennis GJ, James JA, Harley JB. Development of autoantibodies before the clinical onset of systemic lupus erythematosus. N Engl J Med. 2003;349(16):1526-33.

23. Theander E, Jonsson R, Sjostrom B, Brokstad K, Olsson P, Henriksson G. Prediction of Sjogren's syndrome years before diagnosis and identification of patients with early onset and severe disease course by autoantibody profiling. Arthritis Rheumatol. 2015;67(9):2427-36.

24. Wither J, Johnson SR, Liu T, Noamani B, Bonilla D, Lisnevskaia L, Silverman E, Bookman A, Landolt-Marticorena C. Presence of an interferon signature in individuals who are anti-nuclear antibody positive lacking a systemic autoimmune rheumatic disease diagnosis. Arthritis Res Ther. 2017;19(1):41.

25. Bodolay E, Csiki Z, Szekanecz Z, Ben T, Kiss E, Zeher M, Szucs G, Danko K Szegedi G. Five-year follow-up of 665 Hungarian patients with undifferentiated connective tissue disease (UCTD). Clin Exp Rheumatol. 2003;21(3):313-20.

26. Cavazzana I, Franceschini F, Belfiore N, Quinzanini M, Caporali R, CalzavaraPinton P, Bettoni L, Brucato A, Cattaneo R, Montecucco C. Undifferentiated connective tissue disease with antibodies to Ro/SSa: clinical features and follow-up of 148 patients. Clin Exp Rheumatol. 2001;19(4):403-9.

27. Mosca M, Baldini C, Bombardieri S. Undifferentiated connective tissue diseases in 2004. Clin Exp Rheumatol. 2004;22(3 Suppl 33):S14-8.
28. Lu R, Munroe ME, Guthridge JM, Bean KM, Fife DA, Chen H, Slight-Webb SR, Keith MP, Harley JB, James JA. Dysregulation of innate and adaptive serum mediators precedes systemic lupus erythematosus classification and improves prognostic accuracy of autoantibodies. J Autoimmun. 2016;74:182-93.

29. Munroe ME, Lu R, Zhao YD, Fife DA, Robertson JM, Guthridge JM, Niewold TB, Tsokos GC, Keith MP, Harley JB, et al. Altered type II interferon precedes autoantibody accrual and elevated type I interferon activity prior to systemic lupus erythematosus classification. Ann Rheum Dis. 2016;75(11):2014-21.

30. Cossu M, van Bon L, Preti C, Rossato M, Beretta L, Radstake T. Earliest phase of systemic sclerosis typified by increased levels of inflammatory proteins in the serum. Arthritis Rheumatol. 2017;69(12):2359-69.

31. Hochberg MC. Updating the American College of Rheumatology revised criteria for the classification of systemic lupus erythematosus. Arthritis Rheum. 1997;40(9):1725.

32. van den Hoogen F, Khanna D, Fransen J, Johnson SR, Baron M, Tyndall A, Matucci-Cerinic M, Naden RP, Medsger TA Jr, Carreira PE, et al. 2013 classification criteria for systemic sclerosis: an American college of rheumatology/European league against rheumatism collaborative initiative. Ann Rheum Dis. 2013;72(11):1747-55.

33. Vitali C, Bombardieri S, Jonsson R, Moutsopoulos HM, Alexander EL, Carsons SE, Daniels TE, Fox PC, Fox RI, Kassan SS, et al. Classification criteria for Sjogren's syndrome: a revised version of the European criteria proposed by the American-European Consensus Group. Ann Rheum Dis. 2002;61 (6):554-8.

34. Yellen SB, Cella DF, Webster K, Blendowski C, Kaplan E. Measuring fatigue and other anemia-related symptoms with the Functional Assessment of Cancer Therapy (FACT) measurement system. J Pain Symptom Manag. 1997; 13(2):63-74.

35. Wolfe F, Clauw DJ, Fitzcharles MA, Goldenberg DL, Katz RS, Mease P, Russell AS, Russell IJ, Winfield JB, Yunus MB. The American College of Rheumatology preliminary diagnostic criteria for fibromyalgia and measurement of symptom severity. Arthritis Care Res (Hoboken). 2010; 62(5):600-10.

36. Wolfe F, Clauw DJ, Fitzcharles MA, Goldenberg DL, Hauser W, Katz RS, Mease P, Russell AS, Russell IJ, Winfield JB. Fibromyalgia criteria and severity scales for clinical and epidemiological studies: a modification of the ACR Preliminary Diagnostic Criteria for Fibromyalgia. J Rheumatol. 2011;38(6):1113-22.

37. Patterson AJ, Brown WJ, Powers JR, Roberts DC. Iron deficiency, general health and fatigue: results from the Australian Longitudinal Study on Women's Health. Qual Life Res. 2000;9(5):491-7.

38. An JH, Kim YJ, Kim KJ, Kim SH, Kim NH, Kim HY, Kim NH, Choi KM, Baik SH, Choi DS, et al. L-carnitine supplementation for the management of fatigue in patients with hypothyroidism on levothyroxine treatment: a randomized, double-blind, placebo-controlled trial. Endocr J. 2016;63(10):885-95.

39. Daniels J, Brigden A, Kacorova A. Anxiety and depression in chronic fatigue syndrome/myalgic encephalomyelitis (CFS/ME): examining the incidence of health anxiety in CFS/ME. Psychol Psychother. 2017;90(3):502-9.

40. Rinehart J, Hersh E, Issell B, Triozzi P, Buhles W, Neidhart J. Phase 1 trial of recombinant human interleukin-1 beta (rhlL-1 beta), carboplatin, and etoposide in patients with solid cancers: Southwest Oncology, Group Study 8940. Cancer Investig. 1997;15(5):403-10.

41. Rohleder N, Aringer M, Boentert M. Role of interleukin- 6 in stress, sleep, and fatigue. Ann N Y Acad Sci. 2012;1261:88-96.

42. Moreland LW, Genovese MC, Sato R, Singh A. Effect of etanercept on fatigue in patients with recent or established rheumatoid arthritis. Arthritis Rheum. 2006;55(2):287-93.

43. Goligher EC, Pouchot J, Brant R, Kherani RB, Avina-Zubieta JA, Lacaille D, Lehman AJ, Ensworth S, Kopec J, Esdaile JM, et al. Minimal clinically important difference for 7 measures of fatigue in patients with systemic lupus erythematosus. J Rheumatol. 2008;35(4):635-42.

44. Staud R. Are patients with systemic lupus erythematosus at increased risk for fibromyalgia? Curr Rheumatol Rep. 2006;8(6):430-5.

45. Gudbjornsson B, Broman JE, Hetta J, Hallgren R. Sleep disturbances in patients with primary Sjogren's syndrome. Br J Rheumatol. 1993;32(12): 1072-6

46. Overman $\mathrm{CL}$, Kool MB, Da Silva JA, Geenen R. The prevalence of severe fatigue in rheumatic diseases: an international study. Clin Rheumatol. 2016; 35(2):409-15.

47. Moon SJ, Kang KY, Kwok SK, Ju JH, Hong YS, Park SH, Jeon CH, Choi ST, Song JS, Min JK. Differences in quality of life determinants according to the presence of fibromyalgia in middle-aged female patients with systemic 
lupus erythematosus: a multicenter, cross-sectional, single-ethnicity cohort. Int J Rheum Dis. 2018;21(6):1173-84.

48. lannuccelli C, Spinelli FR, Guzzo MP, Priori R, Conti F, Ceccarelli F, Pietropaolo M, Olivieri M, Minniti A, Alessandri C, et al. Fatique and widespread pain in systemic lupus erythematosus and Sjogren's syndrome: symptoms of the inflammatory disease or associated fibromyalgia? Clin Exp Rheumatol. 2012;30(6 Suppl 74):117-21.

49. Perrot S, Peixoto M, Dieude P, Hachulla E, Avouac J, Ottaviani S, Allanore Y. Patient phenotypes in fibromyalgia comorbid with systemic sclerosis or rheumatoid arthritis: influence of diagnostic and screening tests. Screening with the FiRST questionnaire, diagnosis with the ACR 1990 and revised ACR 2010 criteria. Clin Exp Rheumatol. 2017;105(3):354235 Suppl.

50. Choi BY, Oh HJ, Lee YJ, Song YW. Prevalence and clinical impact of fibromyalgia in patients with primary Sjogren's syndrome. Clin Exp Rheumatol. 2016;34(2 Suppl 96):S9-13.

51. Schaible HG. Nociceptive neurons detect cytokines in arthritis. Arthritis Res Ther. 2014;16(5):470.

52. Baglaenko Y, Chang NH, Johnson SR, Hafiz W, Manion K, Ferri D, Noamani B, Bonilla $D$, Rusta-Sellehy $S$, Lisnevskaia $L$, et al. The presence of anti-nuclear antibodies alone is associated with changes in B cell activation and T follicular helper cells similar to those in systemic autoimmune rheumatic disease. Arthritis Res Ther. 2018;20(1):264.

53. Md Yus of MY, Psarras A, El-Sherbiny YM, Hensor EMA, Dutton K, UI-Hassan S, Zayat AS, Shalbaf M, Alase A, Wittmann M, et al. Prediction of autoimmune connective tissue disease in an at-risk cohort: prognostic value of a novel two-score system for interferon status. Ann Rheum Dis. 2018;77(10):1432-9.

54. Sandikci SC, Ozbalkan Z. Fatigue in rheumatic diseases. Eur J Rheumatol. 2015;2(3):109-13.

\section{Publisher's Note}

Springer Nature remains neutral with regard to jurisdictional claims in published maps and institutional affiliations.

Ready to submit your research? Choose BMC and benefit from:

- fast, convenient online submission

- thorough peer review by experienced researchers in your field

- rapid publication on acceptance

- support for research data, including large and complex data types

- gold Open Access which fosters wider collaboration and increased citations

- maximum visibility for your research: over $100 \mathrm{M}$ website views per year

At $\mathrm{BMC}$, research is always in progress.

Learn more biomedcentral.com/submissions 